\title{
1. Hodge Structure and Holonomic Systems
}

\author{
By Masaki KAshiwarA and Takahiro KAWAI \\ Research Institute for Mathematical Sciences, \\ Kyoto University \\ (Communicated by Kôsaku YosidA, M. J. A., Jan. 13, 1986)
}

$\S 0$. Introduction. 0.1 . Let $X$ be a compact complex variety of type $C$ in the sense of Fujiki [3], and $X_{0}$ a non-singular Zariski open subset of $X$. Let $\left(H_{z}, F\right)$ be a variation of Hodge structure on $X_{0}$ of weight $w$. The well-known conjecture (e.g. [1]) is that the cohomology group $H^{n}\left(X ;{ }^{\pi} H_{Q}\right)$ has a Hodge structure of weight $n+w$.

0.2. Toward this direction, we gave the affirmative answer in [4] when $X$ is a non-singular Kähler manifold and $S=X \backslash X_{0}$ is a normally crossing hypersurface. It was also given by Cattani, Kaplan and Schmid [2] independently. But, the Hodge filtration of the cohomclogy groups was not given in an algebro-geometric way.

0.3. In this article we announce how to construct the Hodge filtration in an algebraic way.

$\S 1$. The starting point of the construction is the following method.

1.1. Sandwich method. Let $X$ be a topological space, and $\left(K^{*}, F\left(K^{*}\right)\right)$ a filtered complex of sheaves on $X$. Suppose

$$
H^{n}\left(X ; F^{p}\left(K^{*}\right)\right) \longrightarrow H^{n}\left(X ; K^{*}\right) \text { is injective. }
$$

Let $\left(K_{1}^{*}, F\left(K_{1}^{*}\right)\right),\left(K_{2}^{*}, F\left(K_{2}^{*}\right)\right)$ be two filtered complexes and assume that we have filtered morphisms $K_{1} \stackrel{f}{\longrightarrow} K^{\cdot \stackrel{g}{\longrightarrow}} K_{2}$. Now we make the following hypothesis.

(1.2) $K_{1} \stackrel{f}{\longrightarrow} K^{\cdot} \stackrel{g}{\longrightarrow} K_{2}^{*}$ are quasi-isomorphisms forgetting filtrations.

(1.3) $K_{1}^{\cdot} \longrightarrow K_{2}^{*}$ is a filtered quasi-isomorphism.

Then

$$
H^{n}\left(X ; F^{p}\left(K_{1}^{*}\right)\right) \stackrel{\sim}{\longrightarrow} H^{n}\left(X ; F^{p}\left(K^{*}\right)\right) \stackrel{\sim}{\longrightarrow} H^{n}\left(X ; F^{p}\left(K_{2}^{*}\right)\right),
$$

and therefore $H^{n}\left(X ; F^{p}\left(K_{1}^{*}\right)\right)$ gives the same filtration on $H^{n}\left(X ; K^{*}\right)$ as the one coming from $F\left(K^{*}\right)$.

1.2. Let us return to the situation of $\S 0.2$. Then we know that the complex $\mathcal{L}^{\cdot}(H)_{(2)}$ of square-integrable sections is filtered and that it satisfies (1.1). Further the image of the homomorphism gives the Hodge filtration of the intermediate cohomology groups. In order to illustrate our method, we shall show that the sandwich method combined with these fac's gives another proof of a result of Zucker [7] in the one-variable case. We employ the same notations as in $\S 4$ of [7]. We shall construct $K_{1}^{*}$ and $K_{2}^{*}$ as follows :

$$
K^{\cdot}=\mathcal{L}^{\cdot}(H)_{(2)},
$$




$$
\begin{gathered}
K_{1}^{*}=\left(\mathscr{W}_{0}+z C\right) \otimes_{\mathcal{O}} \mathcal{E}^{(0, \cdot)} \longrightarrow\left(\mathscr{W}_{-2}+z C V\right) \frac{d z}{z} \otimes_{\mathcal{O}} \mathcal{E}^{(0, \cdot)} \\
\left.K_{2}^{*}=\mathcal{H}_{\text {omo }_{\mathcal{O}}}\left(\left(\mathscr{W}_{-2}+z C\right)\right) \frac{d z}{z}, \mathscr{D} b^{(1, \cdot)}\right) \longrightarrow \mathcal{I C o m}_{\mathcal{O}}\left(\mathcal{W}_{0}+z C \mathcal{V}, \mathscr{D} b^{(1, \cdot)}\right) .
\end{gathered}
$$

Here $\mathcal{E}$ (resp., $\mathscr{D} b$ ) denotes the sheaf of $C^{\infty}$-functions (resp., distributions). Then we can easily see that we have a sequence of morphisms $K_{1}^{*} \rightarrow K^{*} \rightarrow K_{2}^{\circ}$. Moreover, Dolbeaut's lemma asserts that $K_{1}^{*}$ and $K_{2}^{*}$ are respectively quasiisomorphic to $\mathscr{W}_{0}+z C V \rightarrow W_{-2}+z C V$ and

$$
\operatorname{Hom}_{\mathcal{O}}\left(\left(\mathscr{W}_{-2}+z C \mathcal{V}\right) \frac{d z}{z}, \Omega^{1}\right) \longrightarrow \mathcal{H}_{o m_{\mathcal{O}}}\left(\mathscr{W}_{0}+z C \mathcal{V}, \Omega^{1}\right)
$$

It is also an easy task to show that they are quasi-isomorphic.

1.3. In a several-variables case, the sandwich method works well similarly. However, the complex corresponding to $\mathscr{W}_{0}+z C V \rightarrow W_{-2}+z C V$ is much more complicated. (See $\S 4.3$ below.)

$\S 2$. 2.0. Let us return to the situation of $\S 0.2$. Let $\mathscr{D}_{X}$ be the sheaf of linear differential operators on $X$, and $\mathscr{M}$ the regular holonomic $\mathscr{D}_{X^{-}}$ module such that its de Rham transform $\mathrm{DR}_{X}(\mathcal{M})$ is isomorphic to ${ }^{\pi} H_{c}$. Let $\mathcal{O}_{r}\left(H_{C}\right)$ denote the right canonical extension of $\mathcal{O}_{X_{0}} \otimes H_{C}$, namely, the vector bundle on $X$ characterized as follows: For each vector field $v$ tangent to an irreducible component $Y$ of $X \backslash X_{0}$ such that $v$ acts identically on the normal bundle of $Y, v$ acts on $\mathcal{O}_{r}\left(H_{C}\right)$ and that the eigenvalues $\lambda$ of $v$ as an endomorphism of $\mathcal{O}_{Y} \otimes \mathcal{O}_{r}\left(H_{C}\right)$ satisfy

$$
-1<\operatorname{Re} \lambda \leqq 0 \text {. }
$$

Then Schmid [6] shows that the Hodge filtration $F(H)$ of $\mathcal{O}_{X_{0}} \otimes H_{C}$ extends to the filtration $F\left(\mathcal{O}_{r}(H)\right)$ of $\mathcal{O}_{r}\left(H_{C}\right)$ by vector subbundles. The $\mathscr{D}_{X}$-module $\mathcal{M}$ is generated by $\mathcal{O}_{r}\left(H_{C}\right)$. Let $F(\mathscr{M})$ be the filtration of $\mathcal{M}$ generated by $F\left(\mathcal{O}_{r}(H)\right)$, i.e., $F_{p}(\mathscr{M})=\sum_{k} F_{k}(\mathscr{D}) F^{k-p}\left(\mathcal{O}_{r}(H)\right)$, where $F_{k}(\mathscr{D})$ denotes the sheaf of linear differential operators of order $\leqq k$.

Proposition 1. (0) $F(\mathscr{M}) \cap \mathcal{O}_{r}\left(H_{C}\right)=F\left(\mathcal{O}_{r}(H)\right)$.

(i ) $\operatorname{Gr}^{F}(\mathscr{M})$ is a Cohen-Macaulay $\mathrm{Gr}^{F}(\mathscr{D})$-module.

(ii) $\operatorname{Ext}_{\mathscr{D}}^{\operatorname{dim} X}(\mathscr{M}, \mathscr{D})$ is filtered isomorphic to $\mathscr{M}$ up to an appropriate shift of filtrations.

2.1. Let $\operatorname{DR}_{X}(\mathscr{M})$ be the de Rham complex $\mathscr{M} \rightarrow \Omega_{X}^{1} \otimes_{\text {O}_{X}} \mathscr{M} \rightarrow \Omega_{X}^{2} \otimes_{\text {O}_{X}} \mathscr{M}$ $\rightarrow \cdots$. Then this is filtered by $F_{p}(\mathscr{M}) \rightarrow \Omega_{X}^{1} \otimes_{\mathcal{O}_{X}} F_{p+1}(\mathcal{M}) \rightarrow \Omega_{X}^{2} \otimes_{\mathcal{O}_{X}} F_{p+2}(\mathscr{M})$ $\rightarrow \cdots$. Our main theorem is the following

Theorem 1. $H^{n}\left(X ; F^{p}\left(\mathrm{DR}_{X}(\mathscr{M})\right)\right) \rightarrow H^{n}\left(X ; \mathrm{DR}_{X}(\mathscr{M})\right)$ is injective and its image gives the Hodge filtrations of $H^{n}\left(X ;{ }^{\pi} H_{C}\right) \simeq H^{n}\left(X ; \mathrm{DR}_{X}(\mathscr{M})\right)$.

§3. 3.0. Let $X$ be a complex analytic space and let $G_{1}$ and $G_{2}$ be $\mathcal{O}_{X^{-}}$ modules. A formal differential homomorphism $f: G_{1} \rightarrow G_{2}$ is, by definition, a $C$-linear sheaf homomorphism such that there locally exists $m$ such that $\left(\operatorname{ad} a_{0}\right)\left(\operatorname{ad} a_{1}\right) \cdots\left(\operatorname{ad} a_{m}\right) f=0$ for any $a_{0}, a_{1}, \cdots, a_{m} \in \mathcal{O}_{X} . \quad$ Here $(\operatorname{ad} a) f=a f-$ fa. A differential homomorphism is a formal differential homomorphism $f: G_{1} \rightarrow G_{2}$ such that $f$ can be extended to an $\mathcal{O}_{s}$-linear homomorphism $f_{S}: \mathcal{O}_{S \times X} \otimes_{\mathcal{O}_{X}} G_{1} \rightarrow \mathcal{O}_{S \times X} \otimes_{\mathcal{O}_{X}} G_{2}$, functorially in $S$. Let us assume that $G_{j}$ is 
filtered by $\mathcal{O}_{X}$-modules $F_{p}\left(G_{j}\right)$ which satisfy

$$
F_{p}\left(G_{j}\right)=0 \text { for } p \ll 0 \text { and } G_{j}=\cup_{p} F_{p}\left(G_{j}\right) \text {. }
$$

We say that $f: G_{1} \rightarrow G_{2}$ is a filtered differential homomorphism if for any $m$ and $a_{1}, \cdots, a_{m} \in \mathcal{O}_{X}$ we have $\left(\operatorname{ad} a_{1}\right) \cdots\left(\operatorname{ad} a_{m}\right) F_{p}\left(G_{1}\right) \subset F_{p-m}\left(G_{2}\right)$. Considering such a filtered homomorphism, we can construct, in an evident way, the filtered derived category. We shall denote it by $\mathrm{DF}(X)$.

3.1. Now, let us suppose that $X$ is smooth. A filtered $\mathscr{D}_{X}$-module is, by definition, a $\mathscr{D}_{X}$-module $\mathscr{M}$ with filteration $F(\mathscr{M})$ satisfying (3.1) and $F_{k}(\mathscr{D}) F_{p}(\mathscr{M}) \subset F_{p+k}(\mathscr{M})$.

Then filtered $\mathscr{D}_{X}$-modules form a derived category.

Proposition 2. $\mathrm{DF}(X)$ is equivalent to the derived category of filtered $\mathscr{D}_{X}$-modules.

The isomorphism from the derived category of filtered $\mathscr{D}_{X}$-modules to $\mathrm{DF}(X)$ is given by the de Rham functor $\mathrm{DR}_{X}$.

3.2. Let us return to the situation of $\S 0.2$ and $\S 2.0$. Let $\mathcal{O}_{l}\left(H_{c}\right)$ denote the left canonical extension of $\mathcal{O}_{x_{0}} \otimes H_{C}$, i.e., the vector bundle obtained similarly to $\mathcal{O}_{r}\left(H_{C}\right)$ in $\S 2.0$ by replacing the condition (2.1) by $0 \leqq \operatorname{Re} \lambda<1$. (Note that $\mathcal{O}_{l}\left(H_{C}\right) \subset \mathcal{O}_{r}\left(H_{C}\right)$ holds by their definitions.) Let $\Omega_{\text {log }}\left(H_{C}\right)$ be the complex

$$
\mathcal{O}_{l}\left(H_{C}\right) \longrightarrow \Omega^{1}(\log S) \otimes \mathcal{O}_{l}\left(H_{C}\right) \longrightarrow \Omega^{2}(\log S) \otimes \mathcal{O}_{l}\left(H_{C}\right) \longrightarrow \cdots
$$

and define $\Omega_{\log }(\mathscr{M})$ by $\Omega_{\log }\left(H_{C}\right) \cap \mathrm{DR}_{X}(\mathscr{M})$. Then the filtration of $\mathcal{O}_{l}\left(H_{C}\right)$ and the filtration of $\mathrm{DR}_{X}(\mathscr{M})$ induce the same filtration on $\Omega_{\log }(\mathscr{M})$. With this filtration, we have

Proposition 3. $\mathrm{DR}_{X}(\mathscr{M})$ is filtered quasi-isomorphic to $\Omega_{\text {iog }}(\mathscr{M})$. In particular $H^{n}\left(X ; F\left(\Omega_{\log }(\mathscr{M})\right)\right)$ gives the Hodge filtrations of $H^{n}\left(X ;{ }^{\pi} H_{C}\right)$.

$\S 4$. We now describe how to construct $K_{1}^{*}$ and $K_{2}^{*}$.

4.1. Let $S$ be a normally crossing hypersurface of a complex manifold $X$, and $\left\{X_{i}\right\}_{i \in I}$ the decomposition into irreducible components of $S$. For a finite subset $\alpha$ of $I$, we set $X_{\alpha}=\bigcap_{i \in \alpha} X_{i}$. Let $V$ be a vector bundle on $X$ and let $V_{\alpha}$ be a vector subbundle of $\left.V\right|_{X_{\alpha}}$ such that $\left.V_{\alpha}\right|_{X_{\beta}} \supset V_{\beta}$ for $\beta \supset \alpha$. Then we denote by $\mathcal{L}\left(\left\{V_{\alpha}\right\}\right)$ the $\mathcal{O}_{X}$-submodule of $V$ consisting of sections $u$ such that $\left.u\right|_{X_{\alpha}} \in V_{\alpha}$. Then $\mathcal{L}\left(\left\{V_{\alpha}\right\}\right)$ is exact in $\left\{V, V_{\alpha}(\alpha \subset I)\right\}$.

4.2. Let us return to the situation in $\S 0.2$ and use the same notations as in the preceding paragraph. Let $M_{i}$ be the monodromy of $H_{R}$ around $X_{i}$ and set $N_{i}=(1 / 2 \pi m \sqrt{-1}) \log M_{i}^{m}$ for some $m \geqq 1$ such that $M_{i}^{m}$ is unipotent. Then $N_{i} \in$ End $\left(\left.\mathcal{O}_{l}(H)\right|_{X_{i}}\right)$. For $\alpha \subset I$ and $t_{i}>0(i \in \alpha)$, we denote by $W(\alpha)$ the $\left(\sum_{i \in \alpha} t_{i} N_{i}\right)$-filtration of $\left.\mathcal{O}_{l}(H)\right|_{X_{\alpha}}$.

4.3. For $\alpha \subset I$, we denote by $\Omega(\alpha)$ the exterior $\mathcal{O}_{X}$-subalgebra of $\Omega(\log S)$ generated by $\Omega^{1}\left(\log X_{i}\right)$ with $i \in \alpha$. Let us now consider the following differential complex $\mathcal{L}^{\cdot}$ :

which is a subcomplex of

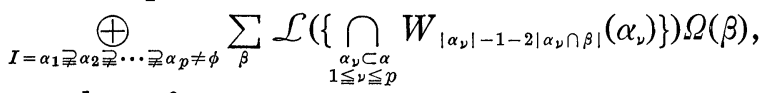

$$
\underset{I=\alpha_{1} \supsetneq \alpha_{2} \underset{\cdots}{*} \cdots \alpha_{p}=\phi}{\bigoplus} \Omega(\log S) \otimes \mathcal{O}_{l}(H) .
$$


The last one is the simple complex associated with the double complex whose first differentiation is the exterior derivative and whose second differentiation is the differential associated with the simplicial complex whose simplex is $\left\{\alpha_{2}, \cdots, \alpha_{p}\right\}$.

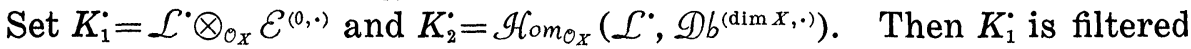
quasi-isomorphic to $\mathcal{L}^{*}$ and $\mathcal{L}^{*}$ is filtered quasi-isomorphic to $\mathrm{DR}(\mathscr{M})$. Moreover $K_{2}^{*}$ is quasi-isomorphic to $R \mathscr{H}$ mo $_{\mathcal{O}_{X}}\left(\mathcal{L}^{*}, \Omega_{X}^{\mathrm{dim}}{ }^{X}\right)$. Then Proposition 1 (ii) implies $K_{2}^{*} \simeq \mathrm{DR}(\mathscr{M})$. Here we used the fact that $\mathcal{E}$ is flat over $\mathcal{O}_{X}$ and the stalk of $\mathscr{D} b$ is injective over $\mathcal{O}_{x}$. (See e.g. [5].) Since we can construct a filtered homomorphism $K_{1} \rightarrow \mathcal{L}^{*}(H)_{(2)}$ and hence another filtered homomorphism $\mathcal{L}^{\cdot}(H)_{(2)} \rightarrow K_{2}^{*}$ by the duality, we can use the sandwich method and obtain Theorem 1.

4.4. In the two-dimensional case, $\mathcal{L}^{*} \simeq \mathrm{DR}(\mathscr{M})$ implies, in particular, that the following complex

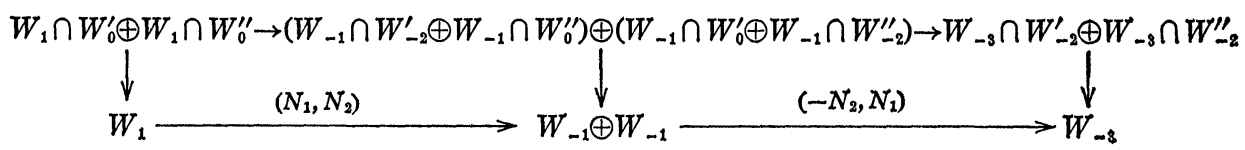

is quasi-isomorphic to $H \rightarrow \operatorname{Im} N_{1} \oplus \operatorname{Im} N_{2} \rightarrow \operatorname{Im} N_{1} N_{2}$, where $W=W\left(N_{1}, N_{2}\right)$, $W^{\prime}=W\left(N_{1}\right)$ and $W^{\prime \prime}=W\left(N_{2}\right)$.

\section{References}

[1] Brylinski, J-L.: (Co)-homologie d'intersection et faisceaux pervers (Sém. Bourbaki, no. 585). Astérisque, 92/93, 129-157 (1982).

[2] Cattani, E., A. Kaplan and W. Schmid: $L^{2}$ and intersection cohomologies for a polarized variation of Hodge structure (preprint).

[3] Fujiki, A.: Closedness of the Douady spaces of compact Kähler spaces. Publ. RIMS, Kyoto Univ., 14, 1-52 (1978).

[4] Kashiwara, M. and T. Kawai: The Poincaré lemma for a variation of polarized Hodge structure. Proc. Japan Acad., 61A, 164-167 (1985).

[5] Malgrange, B.: Ideals of Differentiable Functions. Oxford University Press (1966).

[6] Schmid, W.: Variation of Hodge structure: the singularities of the period mapping. Inv. Math., 22, 211-319 (1973).

[7] Zucker, S.: Hodge theory with degenerating coefficients: $L_{2}$ cohomology in the Poincaré metric. Ann. of Math., 109, 415-476 (1979). 